\title{
Indomethacin Metabolism in Isolated Neonatal and Fetal Rabbit Hepatocytes
}

\author{
M. A. EVANS, C. PAPAZAFIRATOU, R. BHAT, AND D. VIDYASAGAR ${ }^{(29)}$ \\ Department of Pediatrics, Abraham Lincoln School of Medicine, and Department of Pharmacology, School of Basic \\ Medical Science, University of Illinois Medical Center, Chicago, Illinois, USA
}

\begin{abstract}
Summary
Metabolism of indomethacin was examined in freshly isolatèd hepatocytes prepared by liver collagenase perfusion of fetal (28) and neonatal rabbits of $3,5,10,12$, and 25 days of age. Initial cell viability was more than $90 \%$ and linear rates of metabolism were observed for up to $2 \mathrm{hr}$ of incubation. Deacylation of indomethacin to desbenzoyl indomethacin showed a rapid increase early in postnatal development while microsomal $O$-demethylation to desmethyl indomethacin was increased significantly only in the hepatocytes from 25-day-old rabbits. Glucuronide conjugates of indomethacin and indomethacin metabolites accounted for less than $8 \%$ of the total metabolites in hepatocytes from 25-day-old rabbits and less than $2 \%$ in the 3- to 5-day-old rabbits. The maturational development of indomethacin in metabolism may account for previously reported gestational dependent half-life of the drug in the premature infant (9). However, the factors that regulate development of indomethacin metabolism appear to be under more than one control system.
\end{abstract}

\section{Speculation}

The noted adverse effects from indomethacin in the premature infant suffering from patent ductus arteriosus probably results from drug accumulation. Several independent pathways of metabolism for indomethacin have been demonstrated and the differences in their postnatal maturation may cause considerable variation in the patient disposition of the drug. These variations are important in the consideration of drug therapy in the premature infant.

Biologic immaturity and its influence on the varying disposition of drugs is a major source of concern in the treatment of neonates and premature infants. Despite only limited knowledge in this area an increasing number of drugs are being used in preterm and term infants for various cardiac and respiratory problems. Hence, it has become increasingly important to identify those mechanisms which modulate drug disposition during development to help provide better prediction of pharmacologic disposition of a given drug. Hepatocytes isolated by the collagenase perfusion method have been shown to be a useful addition to the methods for study of drug metabolism and toxicity. Substrates for microsomal monooxygenase are actively metabolized in the hepatocyte without added cofactors and detoxification pathways are also functional (14). Thus the isolated hepatocyte provides an integrated cellular model for study of drug metabolism since it retains the structural and functional properties of the intact cell. Billings et al. (4) demonstrated that metabolic rates for selected chemicals in isolated hepatocytes correlate well with in vivo rates of drug metabolism. Other investigators have demonstrated that the isolated hepatocyte is an excellent model to study early stages of hepatotoxicity and allows for determination of structure activity relation- ships (19). This model for study of drug disposition thus compliments others such as the isolated perfused liver and purified enzymes systems which can provide information on, respectively, the role of hepatic blood flow, or the possible formation of reactive intermediates in drug disposition and toxicity.

Indomethacin is being widely used in neonates as an alternative to surgical ligation of the ductus in the treatment of patent ductus arteriosus $(11,13,17)$. However, its use has also been associated with several adverse reactions including reduced renal function and hemorrhagic episodes $(6,5,12)$. Despite the increased usage, little is known about the metabolism of the drug in infants $(9,16$, $18,24,25)$. A recent study demonstrated that the elimination halflife of indomethacin (I) was considerably prolonged as compared to reported adults values and plasma clearance values were approximately $25 \%$ of the adult values (9). In addition plasma halflife in preterm infants correlated inversely with gestational age. Plasma half-life of indomethacin in infants less than 32 wk gestation was significantly prolonged compared to values obtained from infants with gestational ages greater than 33 wk. It was postulated that the decreased clearance observed in the less mature subjects could reflect the immaturity of microsomal enzymes in metabolism of the drug to its inactive metabolites.

Metabolism of I is critical to the total clearance of the drug from the body. Duggan et al. (7) demonstrated that only minimal amounts of I administered to adults are excreted unchanged and that the primary urine metabolites consisted of the conjugated and unconjugated desmethyl indomethacin (DMI) and deschlorobenzoyl indomethacin (DBI). DMI is formed by demethylation through the cytochrome P-450 microsomal pathway while DBI is formed by nonmicrosomal deacylation (Fig. 1). A method for viable preparation of isolated fetal rabbit hepatocyte based on perfusion of the liver with a collagenase containing buffer was developed to examine the quantitative and qualitative aspects of drug metabolism and disposition of drug during development. This study was designed to explore the possibility that maturaticn of hepatic metabolism with increasing postnatal age may be an important factor in disposition of 1 .

\section{MATERIALS AND METHODS}

\section{ANIMALS AND CHEMICALS}

New Zealand rabbits with a mean gestation of 31 days were used in the study. Standard chemicals for the isolation of hepatocytes along with collagenase were obtained from Sigma Chemical Company (St. Louis, MO). Gelatin was obtained from Difco Laboratories (Detroit, MI). Radiolabeled $2-{ }^{14} \mathrm{C}$ I was obtained from New England Nuclear (Boston, MA) and unlabeled metabolites of I were a gift of Merck Sharp and Dohme (Rahway, NJ). Materials for thin layer chromotography (TLC) were purchased from Brinkman (Westbury, NY). 


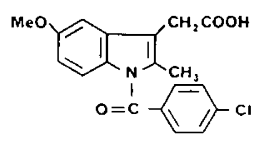

\section{Indomethacin}

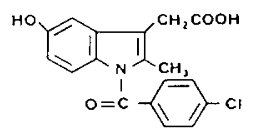<smiles>COc1ccc2[nH]c(C)c(CC(=O)O)c2c1</smiles>

DMI $\mathrm{DBI}$

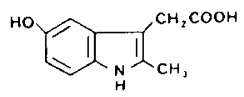

\section{DMDBI}

Fig. 1. Structural formula of I and its major metabolites DMI, DBI, and DMDBI.

\section{ISOLATION OF HEPATOCYTES}

Hepatocytes were isolated by perfusion of the liver with collagenase, using a modified method of Zahlten and Stratman (27). Neonates were lightly anesthetized with ether in a $\mathrm{O}_{2} / \mathrm{CO}_{2}(95 / 5)$ atmosphere before incision. Fetal rabbits used in the study were delivered by Caesarian section using ether in $\mathrm{O}_{2} / \mathrm{CO}_{2}$ atmosphere for anesthesia. The liver was cannulated through the right atrium of the heart into the inferior vena cava. Immediately after cannulation, the portal vein was sectioned and the reverse perfusion was maintained in situ. Flow rate and temperature of the perfusion were $12 \mathrm{ml} / \mathrm{min} / \mathrm{g}$ liver weight and $37^{\circ} \mathrm{C}$, respectively, measured at the cannula. The initial perfusate consisted of calcium free Krebs-Improved Ringers I solution. This media was replaced after 6 min with Krebs-Improved Ringers I media containing $5 \mathrm{mM}$ calcium and $0.5 \mathrm{mg} / \mathrm{ml}$ of collagenase. Both media were kept in equilibrium with $\mathrm{O}_{2} / \mathrm{CO}_{2}(95 / 5, \mathrm{v} / \mathrm{v})$ and buffered to $\mathrm{pH} 7.6$. The perfusion was continued for 10 to $20 \mathrm{~min}$ or until major changes in the liver architecture were observed. The liver was then removed from the animal, transferred to a plastic beaker containing $100 \mathrm{ml}$ of perfusion media with $\mathrm{Ca}^{++}$and collagenase at $37^{\circ} \mathrm{C}$, and gently minced with scissors. After a 12-min incubation period, the cells were filtered through cheese cloth and then separated from debris by centrifugation at $50 \times g$ for $2 \mathrm{~min}(2 \times)$. The cells were resuspended in fresh incubation media at $37^{\circ} \mathrm{C}$ and maintained in $\mathrm{O}_{2} / \mathrm{CO}_{2}$ atmosphere for $30 \mathrm{~min}$ before use.

All incubations were maintained in atmosphere of $\mathrm{O}_{2} / \mathrm{CO}_{2}(95 /$ $5)$, at $37^{\circ} \mathrm{C}$ in plastic flasks with gentle shaking. The basic incubation media consisted of Krebs-Henseleit buffer (pH 7.6) with $1.5 \%$ gelatin and $5 \mathrm{mM}$ calcium. Methionine was included at a 1 $\mathrm{mM}$ final concentration. Initial cell viability, determined by trypan blue exclusion at the end of the 30 -min preincubation, was greater than $90 \%$ for all studies. Cell viability for each experiment was assessed by trypan blue exclusion, succinate stimulation of oxygen uptake, and release of transaminase enzyme into the media (19). Trypan blue exclusion for viability determination was made using $0.2 \%$ trypan blue in Krebs-Henseleit buffer with an improved Neubauer counting chamber. The exclusion assay for each time point was conducted in duplicate using each of the $1 \times 1 \times 0.1$ $\mathrm{mm}$ fields of the counting chamber. Oxygen uptake by the cells was measured with a Clark Oxygen electrode at a polarizing voltage of $-0.6 \mathrm{~V}$ at $37^{\circ} \mathrm{C}$ in the absence and presence of sodium succinate $(10 \mathrm{mM})$. Cellular release of glutamic-pyruvate transaminase into the media was measured by the method of Reitman and Frankel (22). Incubation media $(0.2 \mathrm{ml})$ containing cells was centrifuged for $30 \mathrm{sec}$ in a 1.5-ml Eppendorf test tube containing $0.1 \mathrm{ml}$ of a Dow-Corning silicone oil mixture consisting of eight parts 702 diffusion pump oil and two parts no. 200 fluid (65 centistokes). After separation of the cells from the media $20 \mu \mathrm{l}$ of the media was removed for glutamic-pyruvate transaminase analysis.

\section{INDOMETHACIN METABOLISM}

Metabolism of indomethacin by the isolated hepatocytes was measured by TLC using $2-{ }^{14} \mathrm{C}$ I. Radiolabeled I (New England Nuclear) was mixed with unlabeled drug to a final concentration of $10 \mu \mathrm{Ci} / \mathrm{mMole}$ and then added to isolated fetal rabbit hepatocytes at $0.5 \mathrm{mM}$ final concentration. At time points of $0,15,30$, 60 , and $120 \mathrm{~min}, 1.0 \mathrm{ml}$ of incubation media containing $10^{6}$ cells/ $\mathrm{ml}$ was mixed with an equal volume of $0.2 \mathrm{M}$ acetate buffer $(\mathrm{pH}$ $=5.0$ ) and then extracted with 10 volumes ethyl acetate. After evaporation under nitrogen, the residue was redissolved in $0.1 \mathrm{ml}$ of methanol containing $1 \mathrm{mg} / \mathrm{ml}$ of unlabeled drug and metabolites as carrier in the TLC analysis. Metabolites of indomethacin included DMI, DBI, and desmethyl, deschlorobenzoyl-indometh$\operatorname{acin}$ (DMDBI).

A $50-\mu$ l portion of the methanol was spotted on TLC plates (Brinkman Silica $\mathrm{G}$ ) and developed in an acetic acid/chloroform $(5 / 95)$ solvent system. Retention values for the compounds in the solvent system are: I, 0.35; DBI, 0.24; DMI, 0.09; DMDBI, origin.

The individual compounds were located under ultraviolet light and then removed for liquid scintillation counting. All count per minute (CPM) values from liquid scintillation were converted to disintegration per minute (DPM) based on external standard ratio before calculation. In order to measure conjugated metabolites, the aqueous phase from the initial ethyl acetate extract was incubated with 1000 Fishman units of $\beta$-glucuronidase $(1 \mathrm{mg})$ and $0.5 \mathrm{M}$ acetate buffer $(2 \mathrm{ml}, \mathrm{pH} 5.0)$ for $6 \mathrm{hr}$ at $37^{\circ} \mathrm{C}$. After incubation the samples were extracted and analyzed as described for unconjugated metabolites. Reported values represent the mean value from at least two separate animals with at least four separate analyses/animal at each time point of sample collection. Controls for each experiment were performed using buffer media without cells to account for nonenzymatic breakdown of $\mathrm{I}$.

\section{RESULTS}

Preliminary studies using unlabeled metabolites and high pressure liquid chromatography with fluorescence detection demonstrated that the extraction efficiencies for the unconjugated parent compound and metabolites were (3): I, >98\%; DBI, 95\%; DMI, 91\%; DMDBI, $82 \%$. Complete examination of the TLC plate in initial studies with radiolabeled substrate and isolated cells following development in a 2-dimensional solvent system failed to demonstrate any spots of radioactivity other than I and reported I metabolites. Cell viability by trypan blue analysis was greater than $75 \%$ at the end of all reported studies and only minor $(<5 \%)$ succinate stimulated oxygen uptake could be demonstrated in the freshly isolated hepatocytes. Glutamic-pyruvate transaminase values reflected trypan blue results and were not significantly increased with time.

\section{UNCONJUGATED METABOLITES}

Linear rates of both DBI and DMI formation were observed at each of the gestational ages. Figures 2 and 3 illustrate the increase with time of DBI and DMI in hepatocytes isolated from day 10 and day 12 neonate rabbit. DBI values in hepatocytes from rabbits of early ages were approximately 8-fold greater than DMI and 20fold greater than DMDBI. Since similar linear production of DBI and DMI with time was observed at the other gestational ages, least square regression analysis was used to determine the rate of DBI and DMI formation $/ 10^{6}$ cells. Formation of DMDBI was 


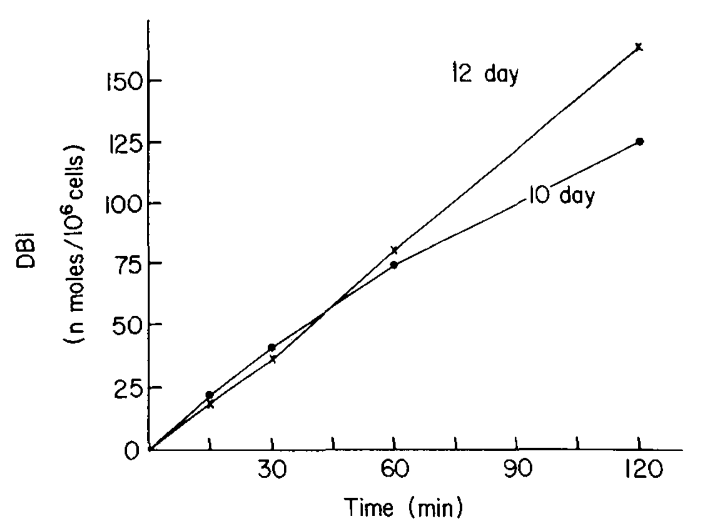

Fig. 2. Graph of the relationship of DBI formation from I with time by freshly isolated hepatocytes obtained from 10- and 12-day-old rabbits.

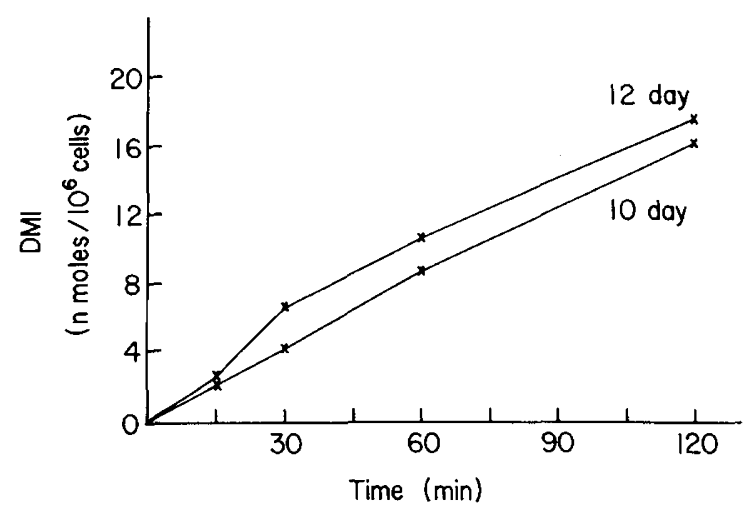

Fig. 3. Graph of the relationship of DMI formation from I with time by freshly isolated hepatocytes obtained from 10- and 12-day-old rabbits.

variable with time and represented less than $5 \%$ of the total metabolites formed. Figure 4 illustrates the increase in total metabolites (conjugated and unconjugated) of $I$ and the decrease in total substrate in hepatocytes isolated from day 10 neonate rabbit. No significant difference was observed between the decrease in I substrate and the increase in total metabolites. Similar results were observed in hepatocytes isolated from rabbits of the other ages. Gestational and postnatal development of DBI and DMI metabolic pathways are illustrated in Figure 5 which shows the rate of DBI and DMI formation as nmoles $/ 10^{6}$ cells $/ \mathrm{min}$ based on least squares regression analysis of results from the individual experiments, for each of the ages studied. Deacylation of I to DBI showed a very early increase with gestational age and was significantly increased above fetal activity within three days of postnatal life. $O$-demethylation of I to DMI, however, showed a much slower postgestational development and was not significantly increased in activity until after day 12 of postnatal life.

\section{CONJUGATED METABOLITES}

Initial studies to detect sulfate ester conjugation using purified sulfatase enzyme, failed to demonstrate the presence of any sulfated I or I metabolites in incubations with hepatocytes from day 28 (fetal), day 3 , or day 25 rabbits. Table 1 shows the total amount of the individual glucuronidated metabolites at each of the gestational ages formed by hepatocytes after $60 \mathrm{~min}$ of incubation. Although a postgestational development could be demonstrated in the rate of conjugation, less than $8 \%$ of total metabolites were conjugated in hepatocytes from day 25 rabbits. Little to no conjugating activity with I or I metabolites could be observed in hepatocytes from fetal rabbits. Examinations of the individual metabolites from hepatocytes of day 25 rabbits revealed that a significant proportion $(<15 \%)$ of total DMI and DMDBI were present as a glucuronide conjugate, while less than $1 \%$ of DBI was glucuronidated. Summation of the individual conjugated and

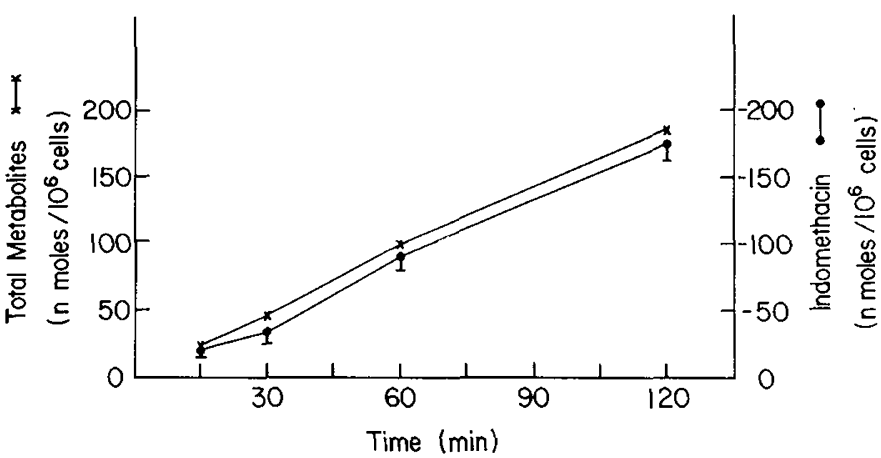

Fig. 4. Comparison of the increase in total I metabolites with the decrease in total I substrate in freshly isolated hepatocytes from 10-dayold rabbits.

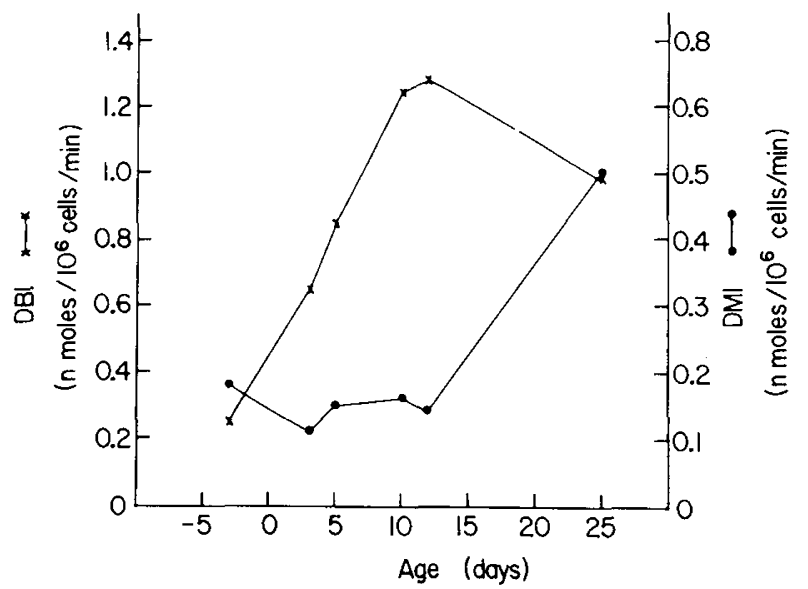

Fig. 5. Development of DBI and DMI metabolic pathways with age in freshly isolated rabbit hepatocytes.

Table 1. Effect of gestational age on formation of glucuronide conjugated metabolites

\begin{tabular}{lcccc}
\hline \multirow{2}{*}{ Age } & \multicolumn{4}{c}{ Conjugated metabolites (nmoles $/ 10^{6}$ cells) } \\
\cline { 2 - 5 } & I & DBI & DMI & DMDBI \\
\hline Fetus (day 28) & 0.72 & 0.11 & 1.06 & 0.41 \\
Day 3-5 & $<0.1$ & 0.22 & 1.12 & 1.00 \\
Day 10-12 & $<0.1$ & 0.28 & 1.23 & 0.46 \\
Day 25 & 3.08 & 0.80 & 4.71 & 1.22 \\
\hline
\end{tabular}

${ }^{1}$ Each value represents the mean from two experiments, four analyses each after $60 \mathrm{~min}$ of incubation.

unconjugated metabolites showed that oxidative metabolism constituted $35 \%$ of the total metabolites in hepatocytes from both fetal and day 25 rabbits but only $10 \%$ in hepatocytes from 10 - to 12 day old rabbits.

\section{DISCUSSION}

Drug metabolism is a particularly complex biologic function because the components are multienzyme in nature and are dependent on a continuous supply of cofactors which are in turn generated by other multienzyme systems. Because of this complex system, study of the factors that regulate in vivo drug metabolism, such as maturation, is probably better conducted using the intact cell rather than isolated enzyme systems. The main organ for drug biotransformation is the liver and although isolated liver cells from adult animals have been extensively used in the study of drug metabolism no such experiments have been conducted utilizing fetal and neonatal liver cells (15). In this study we demonstrate that isolated hepatocytes obtained from fetal and neonatal rabbits are active in both phase I and II metabolism of I. Linear rates of 
metabolic activity were observed for up to $2 \mathrm{hr}$ of incubation and consistent results were observed between animals within each age group.

I undergoes three separate pathways of metabolism: (1) $O$ demethylation by the microsomal oxidative enzyme, (the cytochrome P-450 system) to form DMI; (2) deacylation by the cytosol enzymes to form DBI and; (3) glucuronidation by the conjugating enzymes of the endoplasmic reticulum. Studies in human premature and full-term newborn infants have demonstrated that there is a progressive increase in the activity of the hepatic microsomal drug metabolizing enzymes during the last trimester of gestation and in the neonatal period (2). At term cytochrome P-450 appears to approach approximately 60 to $70 \%$ of the adult values. However, investigation of hepatic microsomal enzyme preparations in different animal species at term have shown negligible or low levels of the drug metabolizing enzyme activity $(20,21)$. In the rabbit hepatic microsomes two general patterns of age related changes have been demonstrated: (1) a gradual increase in selected microsomal enzymes from 3 days to 1 month and (2) a discontinuous increase with a sharp jump in benzephetamine and benzpyrene hydroxylase activity and cytochrome P-450 content between 2 wk and 1 month of age (10). Results from our study with the isolated hepatocytes are consistent with the above findings by showing a marked increase in the rate of production of DMI after $2 \mathrm{wk}$ of life. However, other investigators have reported that the components of the microsomal drug-oxidizing electron transport chain and the drug-oxidizing capacity in the perinatal rabbit liver have a marked increase in activity during the first 2 wk of life (21). Studies with hepatic microsomes from the guinea pig have also demonstrated a rapid postnatal increase in $O$-demethylation using $p$-nitroanisole as substrate. Maximal activity in microsomal $O$ demethylation in these studies was observed by day 14 of postnatal age (8). The reasons for these reported discrepancies in the rate of ontogensis in drug metabolism activity are unknown but may reflect differences in species, substrates, and methods of microsomal preparation.

In the neonatal rabbit hepatocyte, I was found to be mainly metabolized by nonmicrosomal deacylation to DBI. This is in contrast to the finding of Duggan et al. (7) that in adult rabbits demethylation to DMI followed by extramicrosomal deacylation to DMDBI is the major catabolic pathway, while direct deacylation to $\mathrm{DBI}$ is a secondary competing terminal reaction. A very early developmental increase in DBI formation was observed indicating that the factors which control deacylation mature independently from those which control oxidative metabolism.

Conjugation of I and metabolites of I takes place in the liver and constitute the "major" component of the excreted drug in the adult (26). In our study glucuronide conjugation did not appear to be a major metabolic pathway with $<8 \%$ of the total metabolites conjugated in hepatocytes from day 25 rabbits. However, there is a steady increase in hepatic glucuronidation with advancing age. Several explanations have been proposed to account for the low rate of glucuronidation observed in the perinatal hepatic tissues. These range from the relatively low proportion of hepatic parenchymal cells and reduction of the smooth endoplasmic reticulum in the perinatal liver to the presence of inhibitory factors and repressor factors, as well as differences in enzyme configuration in the newborn (23). Thus the UDP-glucuronyl transferase system appears to be initially deficient or repressed in the newborn and activity develops only gradually during postnatal life. This development appears to be under separate controls from those factors which control oxidative metabolism. Consistent with results from metabolism studies in adults no sulfate conjugates of I or I metabolites were observed in this study.

The freshly isolated hepatocyte provides a unique model for study of selected factors which regulate the in vivo maturation of drug metabolism. Development of this model system for study of I metabolism demonstrated that maturation of drug metabolic pathways is under several independent control systems. Further study on the development of drug metabolism in the neonate using the freshly isolated hepatocytes model will provide impor- tant information on the factors that are rate controlling for these processes in the intact animal.

\section{SUMMARY}

Deacylation and demethylation of $I$ in fetal and newborn rabbits appears to be under separate control systems which demonstrate different gestational developmental patterns. In hepatocytes from day 28 fetal rabbits the rate of DBI and DMI formation was 0.23 and $0.13 \mathrm{nmoles} / 10^{6}$ cells $/ \mathrm{min}$, respectively. DBI formation by the cytosol enzymes demonstrated a very early maturational increase with a maximal value of $1.3 \mathrm{nmole} / 10^{6} \mathrm{cells} / \mathrm{min}$ observed at day 12 of postnatal life. Microsomal $O$-demethylation of I to DMI increased significantly only after day 12 of postnatal life and demonstrated a maximal value of 0.5 nmoles $/ 10^{6}$ cells/ min at day 25 of postnatal life. Less than $8 \%$ of the total metabolites were glucuronidated even in hepatocytes from day 25 rabbits. These results indicate that the gestational related changes in I half-life previously reported in premature infants (9) is probably related to separate distinct patterns in maturation of the drug metabolism enzyme systems.

\section{REFERENCES AND NOTES}

1. Alvan, G., Orina, M., Bertilsson, L., Ekstrand, R., and Palmer, L.: Pharmacokinetics of indomethacin. Clin. Pharmacol. Ther., 18: 364 (1975).

2. Aranda, J. V., MacLeod, S. M., Renton, K. W., and Eade, N. R.: Hepatic microsomal drug oxidation and electron transport in newborn infants. Pediatr. Pharmacol Ther., 85: 534 (1974).

3. Bernstein, M., and Evans, M. A.: Quantitation of indomethacin and metabolites in urine by HPLC-fluorescense detection. J. Chromatogr. Biomed. Appl., (in press).

4. Billings, R. E., McMahon, R. E., Ashmore, J., and Wagle, S. R.: The metabolism of drugs in isolated rat hepatocytes. Drug Metab. Dispos., 5: 518 (1977).

5. Brater, D. C.: Effects of indomethacin on salt and water homeostasis. Clin. Pharmacol. Ther., 25: 322 (1979).

6. Chapman, R. A.: Suspected adverse reactions to indomethacin. Can Med. Assoc., 95: 1156 (1966).

7. Duggan, D. E., Hegans, A. F., Kwan, K. C., and McMahon, F. G.: The metabolism of indomethacin in man. J. Pharmacol. Exp. Ther., 18I: 563 (1972).

8. Ecobichon, D. J., Dykeman, R. W., and Hansell, M. M.: The development of hepatic drug metabolizing enzymes in perinatal guinea pigs: a biochemical and morphological study. Can J. Biochem., 56: 736 (1978).

9. Evans, M. A., Bhat, R., Vidyasagar, D., Vadapalli, M., Fisher, E., and Hastreiter, A.: Gestational age and indómethacin elimination in the neonate. Clin. Pharmacol. Ther., 26: 746 (1979).

10. Fouts, J. R., and Devereux, T. R.: Developmental aspects of hepatic and extrahepatic drug-metabolizing enzyme systems: microsomal enzymes and components in rabbit liver and lung during the first month of life. J. Pharmacol. Exp. Ther., 183: 458 (1972).

11. Friedman, W. F., Hirschklau, M. J., Printz, M. P., Pitlick, P. T., and Kirkpatrick, S. E.: Pharmacologic closure of patent ductus arteriosus in premature infant. N. Engl. J. Med., 295: 526 (1976).

12. Friedman, Z., Whitman, V., Maisels, N. Y., Berman, W., Marks, K. H., and Vesell, E.: Indomethacin disposition and indomethacin induced platelet dysfunction in premature infants. J. Clin. Pharmacol., 18: 272 (1978).

13. Heymann, M. A., Rudolph, A. M., and Silverman, N. H.: Closure of the ductus arteriosus in premature infants by inhibition of prostaglandin synthesis. $\mathrm{N}$. Engl. J. Med., 295: 530 (1976).

14. Jones, D. P., Thor, H., Anderson, B., and Orrenius, S.: Detoxification reaction in isolated hepatocytes. J. Biol. Chem., 253: 6031 (1978).

15. Klinger, W.: Development of drug metabolizing enzymes in drug disposition during development. In: P. L. Monselli: Drug Disposition During Development. p. 7I (Spectrum Publications, New York, 1977)

16. McCarthy, J. S., Zies, L. G., and Gelband, H.: Age dependent closure of the patent ductus arteriosus by indomethacin. Pediatrics, 62: 706 (1978).

17. Merritt, T. A., Disessa, T. G., Feldman, B. H., and Kirkpatrick, S. E.: Closure of the ductus arteriosus with ligation and indomethacin: a consecutive experience. J. Pediatr., 93: 639 (1978).

18. Neal, W. A., Kyle, J. M., and Mullett, M.D.: Failure of indomethacin therapy to induce closure of patent ductus arteriosus in premature infants with respiratory distress syndrome. J. Pediatr., 91: 621 (1977).

19. Orrenius, S., Thor, H., Rajo, J., and Berggren, M.: Isolated rat hepatocytes as an experimental tool in the study of cell injury. Effect of anoxia. Forensic Sci., 8: 255 (1976).

20. Rane, A., Sjovist, F., and Orrenius, S.: Drugs and fetal metabolism. Clin. Pharmacol. Ther., 14: 666 (1972).

21. Rane, A.; Berggren, M., Yaffe, S., and Ericsson, J. L. E.: Oxidative drug metabolism in the perinatal rabbit liver and placenta. A biochemical and morphologic study. Xenobiotica, 3: 37 (1973).

22. Reitman, S., and Frankel, S.: A colormetric method for the determination of serum glutamic oxaloacetic and serum glutamic pyruvic transaminases. Am. J. Clin. Pathol., 28: 56 (1957). 
23. Testa, B., and Jenner, P.: Drug Metabolism. (Marcel Dekker Inc., New York, 1978).

24. Thalji, A. A., Carr, I., Yeh, T. F., Raval, D., Luken, J. A., and Pildes, R. S.: Pharmacokinetics of intravenously administered indomethacin in premature infants. J. Pediatr., 97: 995 (1980).

25. Yaffe, S. J., Friedman, W. F., Rogers, D., Long, P., Ragni, M., and Saccar, C. The disposition of indomethacin in preterm babies. J. Pediatr., 97: 1001 (1980).

26. Yesair, D. W., Callahan, M., Remington, L., and Kensler, C. J.: Role of enterohepatic cycle of indomethacin on its metabolism, distribution in tissues and it excretion by rats, dogs and monkeys. Biochem. Pharmacol., 19: 1579 (1970).

Copyright $@ 1981$ International Pediatric Research Foundation, Inc. $0031-3998 / 81 / 1511-1406 \$ 02.00 / 0$
27. Zahlten, R. N., and Stratman, F. W. The isolation of hormone sensitive rat hepatocytes by a modified enzymatic technique. Arch. Biochem. Biophys., 163. 600 (1974).

28. Presented in part at the National Meeting of the Federation of American Societies for Experimental Biology, April 1980.

29. Requests for reprints should be addressed to: Dr. D. Vidyasagar, Department of Pediatrics University of Illinois at the Medical Center, P.O. Box 6998, Chicago, IL 60680 (USA)

30. This research was supported in part by United States Public Health Service Grant DA 01877 and University of Illinois Grant 2-46-38-69-350 and 7658. 